\title{
Multistability and Clustering in a Population of Synthetic Genetic Oscillators via Phase-Repulsive Cell-to-Cell Communication
}

\author{
Ekkehard Ullner, ${ }^{1}$ Alexei Zaikin, ${ }^{2}$ Evgenii I. Volkov, ${ }^{3}$ and Jordi García-Ojalvo ${ }^{1}$ \\ ${ }^{1}$ Departament de Física i Enginyeria Nuclear, Universitat Politècnica de Catalunya, Colom 11, E-08222 Terrassa, Spain \\ ${ }^{2}$ Department of Mathematics, University of Essex, Wivenhoe Park, Colchester CO4 3SQ, United Kingdom \\ ${ }^{3}$ Department of Theoretical Physics, Lebedev Physical Institute, Leninskii 53, Moscow, Russia
}

(Received 16 April 2007; published 2 October 2007)

\begin{abstract}
We show that phase-repulsive coupling eliminates oscillations in a population of synthetic genetic clocks. For this, we propose an experimentally feasible synthetic genetic network that contains phase repulsively coupled repressilators with broken temporal symmetry. As the coupling strength increases, silencing of oscillations is found to occur via the appearance of an inhomogeneous limit cycle, followed by oscillation death. Two types of oscillation death are observed: For lower couplings, the cells cluster in one of two stationary states of protein expression; for larger couplings, all cells end up in a single (stationary) cellular state. Several multistable regimes are observed along this route to oscillation death.
\end{abstract}

DOI: 10.1103/PhysRevLett.99.148103

Synthetic gene-regulation networks have the potential to enhance our knowledge of cellular processes. Besides applications in the design of specific functions, these systems can encourage the study of the design principles of natural systems, by offering a well-controlled test bed in which the functions of natural gene networks can be isolated and studied in detail, independently of the rest of the cellular machinery [1,2]. A pioneering example of a synthetic genetic circuit is the repressilator, a DNA construct of three transcription factors that repress expression of each other in a circular way. When such a construct is introduced into E. coli, clear-cut oscillations in the expression of all three genes are observed [3].

The repressilator is one of the simplest genetic modules to produce near harmonic oscillations in protein levels. It can be constructed experimentally, and recent evidence indicates that its circuit architecture may be present in natural genetic networks [4]. Given that cells are frequently subject to chemical signals from neighboring cells, it is worth studying the effect of such chemical coupling on the dynamics of populations of genetic oscillators. Modeling studies, for instance, have shown that coupled repressilators can work as a macroscopic genetic clock [5]. But coupling can be devised in different ways in this synthetic system. In contrast to Ref. [5], in what follows, the cell-to-cell communication module is designed to have a repressive and phase-repulsive influence [6-8] on the repressilator oscillations. In biology, phase-repulsive coupling is used, e.g., to explain morphogenesis in Hydra regeneration and animal coat pattern formation [9], neural activity in the brain of songbirds [10], the jamming avoidance response in electrical fish [11], and regulation in the respiratory system [12]. Our results show that this type of coupling leads to many different dynamical regimes, including multistability, cluster formation, and oscillation death. To that end, we have modified the repressilator so that it has a weak relaxatorlike dynamics [13], introducing a temporal asymmetry in the dynamics that reduces the
PACS numbers: 87.18. $-\mathrm{h}, 05.45 .-\mathrm{a}, 87.16 . Y c$

harmonic character of the oscillations. Incidentally, the required modifications take the repressilator closer to more realistic in vivo conditions, since the lifetimes of some of the proteins involved can be longer than in the original repressilator, where degradation tags had to be used to shorten their lifespan [3].

In the original repressilator design [3], the gene lacI (from E. coli) expresses the protein LacI, which inhibits transcription of the gene tetR (from the tetracyclineresistant transposon $\mathrm{Tn}$ 10). The product of the latter, TetR, inhibits transcription of the gene $c I$ (from the $\lambda$ bacteriophage). Finally, the protein product $\mathrm{CI}$ of the gene $c I$ inhibits expression of lacI and completes the cycle (Fig. 1). Cell-to-cell communication is realized by an additional feedback loop involving two other proteins [5,14,15]: LuxI, which produces a small autoinducer (AI) molecule that can diffuse through the cell membrane, and LuxR, which responds to the autoinducer by activating transcription of a second copy of the repressilator gene lacI. Placing the luxI gene under inhibitory control of TetR leads to a positive feedback loop, mediated by intercell coupling, of TetR on itself (Fig. 1). This loop competes with the overall negative feedback loop along the repressilator ring and leads, in contrast to Ref. [5], to a phaserepulsive coupling.

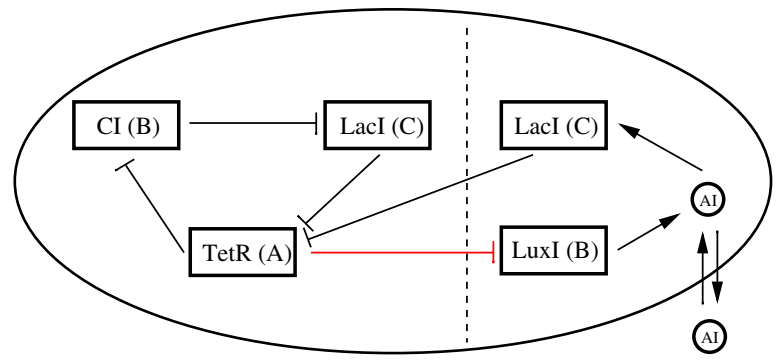

FIG. 1 (color online). Scheme of the modified repressilator with repressive cell-to-cell communication. 
The mRNA dynamics is described by the following Hilltype kinetics, with Hill coefficient $n$ :

$$
\begin{gathered}
\dot{a}_{i}=-a_{i}+\frac{\alpha}{1+C_{i}^{n}}, \\
\dot{b}_{i}=-b_{i}+\frac{\alpha}{1+A_{i}^{n}}, \\
\dot{c}_{i}=-c_{i}+\frac{\alpha}{1+B_{i}^{n}}+\frac{\kappa S_{i}}{1+S_{i}},
\end{gathered}
$$

where the subindex $i$ denotes the cell, and the variables $a_{i}$, $b_{i}$, and $c_{i}$ represent the mRNA concentrations of tetR, $c I$, and lacI, respectively. $\alpha$ is the dimensionless transcription rate of the repressilator-gene promoters in the absence of a repressor (assumed equal), and $\kappa$ is the maximum transcription rate of the LuxR promoter. The protein dynamics is given by

$$
\dot{A}_{i}=\beta_{a}\left(a_{i}-A_{i}\right)
$$

and is given similar for $B_{i}$ (with $b_{i}$ ) and $C_{i}$ with $c_{i} . A_{i}, B_{i}$, and $C_{i}$ denote the concentration of the proteins TetR, CI, and LacI, respectively, and $\beta_{a, b, c}$ are the ratios between mRNA and protein lifetimes (inverse degradation rates). The model is made dimensionless by measuring time in units of the mRNA lifetime (assumed equal for all genes) and the mRNA and protein levels in units of their Michaelis constant. The mRNA concentrations are additionally rescaled by the ratio of their protein degradation and translation rates [5].

A moderate increase of the Hill coefficient to $n=2.6$, a value in agreement with recent experimental measures [16], together with different lifetime ratios $\beta_{a}=0.85$, $\beta_{b}=0.1$, and $\beta_{c}=0.1$, evoke a stronger nonlinear dynamics of the repressilator, leading to the appearance of two time scales in the time series. As an example, Fig. 2 compares the dynamics of CI concentration $(B)$ in the standard repressilator (dashed line) with that resulting from the parameters described above (solid line), in the absence of intercell coupling $(\kappa=0)$. The latter exhibits slow or fast dynamics, with a fast concentration increase and a relative slow decay, whereas the original repressilator exhibits a quasiharmonic dynamics with only one time scale. The parameter values used, given in the caption of Fig. 2, are experimentally reasonable, corresponding to mRNA lifetimes of $5 \mathrm{~min}$, a TetR protein lifetime of $5.9 \mathrm{~min}, \mathrm{a} \mathrm{CI}$ and LacI protein lifetime of $50 \mathrm{~min}$, an AI lifetime of $5 \mathrm{~min}$, Michaelis constants of $20 \mathrm{nM}$, translation rates of 0.1 proteins/s, and an unrepressed transcription rate of 0.4 transcripts/s for $t e t R$ and 0.047 transcripts/s for cI and lacI.

The third term on the right-hand side of Eq. (3) represents activated production of lacI by the autoinducer molecule, whose concentration inside cell $i$ is denoted by $S_{i}$. The dynamics of $C I$ and LuxI can be considered identical, assuming equal lifetimes of the two proteins and given that their production is controlled by the same protein (TetR).

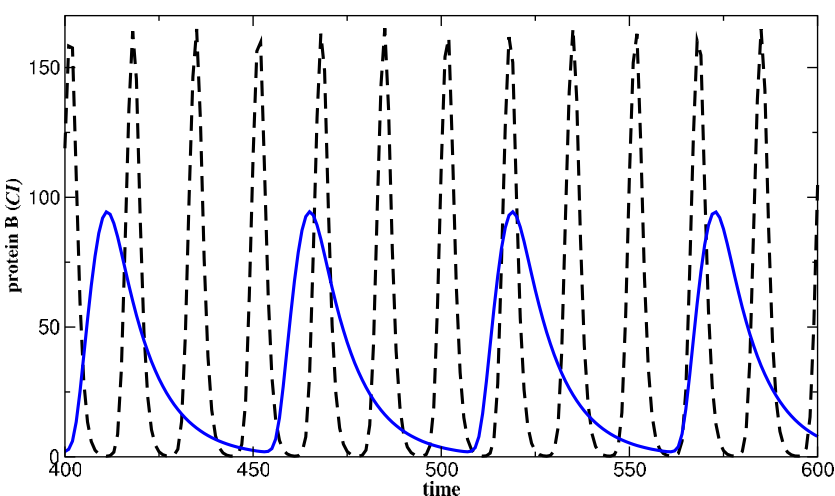

FIG. 2 (color online). The modified uncoupled $(\kappa=0)$ repressilator (solid blue line) with the parameters $n=2.6, \alpha=216$, $\beta_{a}=0.85$, and $\beta_{b, c}=0.1$ compared to the original repressilator (dashed black line) with the parameters $n=2.0, \alpha=216$, and $\beta_{a, b, c}=1.0$.

Hence, the synthesis of the autoinducer $S_{i}$ can be considered to be controlled by the concentration $B_{i}$ of the protein CI. Taking also into account the intracellular degradation of the AI and its diffusion toward or from the intercellular space, the dynamics of $S_{i}$ is given by:

$$
\dot{S}_{i}=-k_{s 0} S_{i}+k_{s 1} B_{i}-\eta\left(S_{i}-S_{e}\right),
$$

where the diffusion coefficient $\eta$ depends on the permeability of the membrane to the autoinducer. The AI concentration $S_{i}$ is scaled by its Michaelis constant [see Eq. (3)]. Because of the fast diffusion of the extracellular AI $\left(S_{e}\right)$ compared to the repressilator period, we can apply the quasi-steady-state approximation to the dynamics of the external AI, which leads to

$$
S_{e}=Q \bar{S} \equiv Q \frac{1}{N} \sum_{i=1}^{N} S_{i}
$$

The parameter $Q$ is defined as $Q=\left(\delta N / V_{\text {ext }}\right) /\left(k_{s e}+\right.$ $\delta N / V_{\text {ext }}$ ) [5], with $N$ the cell number, $V_{\text {ext }}$ the total extracellular volume, $k_{s e}$ the extracellular AI degradation rate, and $\delta$ the product of the membrane permeability and the surface area. We assume $k_{s e} \sim 0.6 \mathrm{~h}^{-1}$, which is reasonable for a medium with $p \mathrm{H} \sim 7$ [15]. The membrane permeability coefficient for the AI corresponds to $10^{-9} \mathrm{~m} / \mathrm{s}$, which is on the order of magnitude of similarly sized biomolecules, such as tryptophan and glucose [17]. The bifurcation parameter $Q$ depends on $N$ in a nonlinear way but can be varied in a controlled way in a chemostat experiment by changing the total chemostat volume. The numerical results that follow do not depend qualitatively on the cell number, and thus we assume below a fixed value of $N$.

The suggested rewiring of the repressilator with the quorum sensing feedback loop leads to competition between the repressilators. The mechanism of this effect includes two repressions: on the one hand, the intracellular repression due to the cyclic inhibition characteristic of the 
repressilator, and, on the other hand, the intercellular repression evoked by the cell-to-cell communication between different repressilators.

We are interested in stable dynamical regimes with a significant basin of attraction, because only those are biologically relevant, given the immanent noise existing in cells $[16,18]$. Dynamical regimes are defined by qualitatively distinct temporal behavior of the mRNAs and proteins in stationary conditions (i.e., after transients have died out). Using $Q$ as bifurcation parameter, we simulate numerically 1000 realizations (for $N=100$ cells) with random initial conditions for each $Q$ value considered and look for the resulting stable dynamical regime. Figure 3 plots the resulting frequency of stable regimes for increasing $Q$. If the system has only one stable regime at a given parameter set, all 1000 initial conditions result in the same regime, but coexistence of more than one stable regime leads to a split of the 1000 initial conditions on several regimes.

The results shown in Fig. 3 reveal a transition from selfoscillations to a single stable fixed point as the coupling $Q$ increases. This transition is gradual and exhibits a multiplicity of regimes. For $Q \lessgtr 0.13$, only self-oscillations are found. The time series depicted in Fig. 4(a) illustrates this regime, which is characterized by large oscillations with the same amplitude and period for all repressilators. The repressive character of the coupling destabilizes the inphase dynamics and leads to a spreading of the phases among all oscillators. After a certain transient time, oscillatory clusters appear [19-21]. The population selforganizes into three clusters of cells that oscillate with a phase difference of $2 \pi / 3$. The separation into three clusters could provide the population of cells with high reliability and stress resistance, because at any given time the

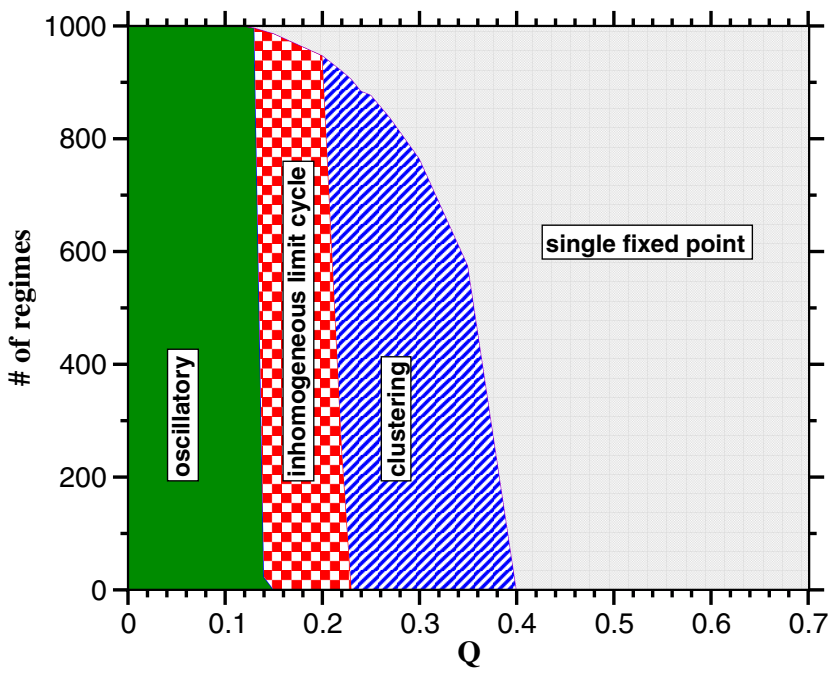

FIG. 3 (color online). Distribution of stable regimes for increasing coupling strength $Q$. Typical time series of each dynamical regime are shown in Fig. 4. The parameters are $N=100, n=2.6, \alpha=216, \beta_{a}=0.85, \beta_{b}=0.1, \beta_{c}=0.1$, $\kappa=25, k_{s 0}=1.0, k_{s 1}=0.01$, and $\eta=2.0$. cells in the different clusters are in different states of the limit cycle, and hence each cluster will be affected differently by sudden environmental stresses such as chemicals or lack of nutrients.

At $Q \approx 0.13$, the self-oscillatory regime disappears abruptly, and a new dynamical regime arises in which some of the cells become trapped in a quasisteady state with a negligible amplitude, while the rest undergo small amplitude oscillations in protein concentration. This dynamical regime corresponds to an inhomogeneous limit cycle (IHLC) and can be observed in detail in Fig. 4(b). Up to now, IHLCs were found only in a model of two coupled Brusselators [22,23]. Here we have found stable IHLCs in a biologically reasonable model of many interacting cells. The system exhibits many possible distributions of cells between the high-concentration, quasisteady-state regime and the low-concentration protein oscillations, but the majority of the repressilators populate the quasiconstant protein level. In the particular case of Fig. 4(b), 95 cells (out of 100) reside in the high-level quasisteady state, while the other 5 are in the low-level oscillating state. In this dynamical regime, cells do not switch from one regime to the other; i.e., there is no mixing of the two populations.

The IHLC shown in Fig. 4(b) is not the only attractor of the system in the range of $Q$ values where it exists. As shown in Fig. 3, a small percentage of the numerical realizations end up in a different attractor, represented by a light gray area on top. In this attractor, all cells exhibit the same stable state of protein concentration, as shown in Fig. 4(d). This single fixed point attractor becomes more likely for larger coupling strengths $Q$.

At $Q \approx 0.2$, a second abrupt transition takes place, through which the inhomogeneous limit cycle disappears, and a regime of fully developed oscillation death arises.
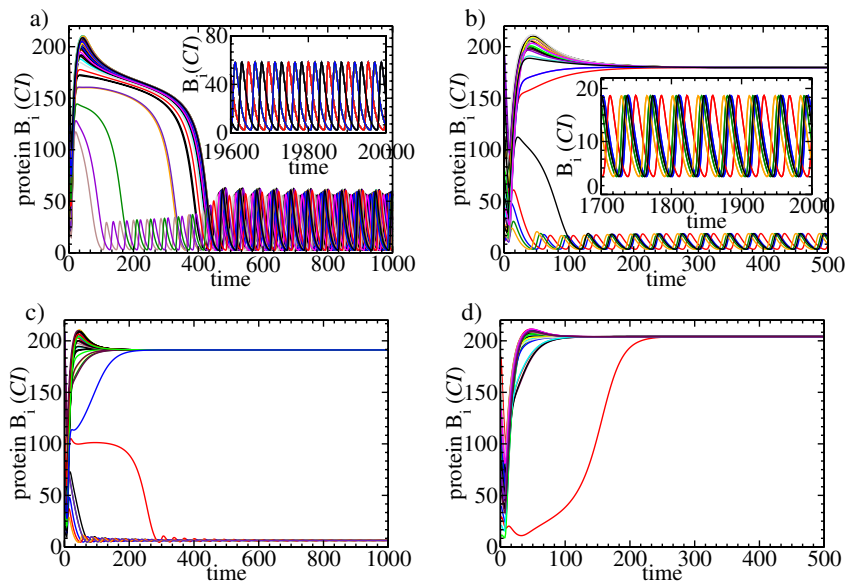

FIG. 4 (color online). The corresponding time series to Fig. 3 with the four different dynamical regimes. (a) $Q=0.11$, oscillatory; (b) $Q=0.16$, inhomogeneous limit cycle; (c) $Q=0.23$, clustering; (d) $Q=0.4$, single fixed point. The insets in (a) and (b) show zoomed parts of the same realization. The other parameters are as in Fig. 3. 
Such behavior has already been reported numerically in a system of coupled genetic relaxator oscillators by Kuznetsov and co-workers [24]. In this regime, shown in Fig. 4(c), all cells stop oscillating, but they do so differentiating into two different clusters. Similarly to the IHLC described above, some cells get stuck in a steady state of high protein expression [94 cells in the case depicted in Fig. 4(c)], while the others exhibit much smaller protein concentrations of the CI protein (6 cells in the case mentioned). Because of the intracellular repression characteristic of the repressilator, a small CI protein production implies a high TetR protein level and vice versa. Since each cluster is specialized in the production of a different protein, this regime could be interpreted as a mechanism of artificial differentiation in an isogenic population of cells. As in the case of the IHLC, cells may distribute into the two clusters at high and low CI levels in many different ratios which differ slightly in the constant protein levels. Hence, in fact many different attractors exist, and a finetuning of protein production can be achieved.

Again, the clustering regime described in the previous paragraph coexists with the single fixed point attractor [Fig. 4(d)], which becomes increasingly more likely for increasing $Q$, until it turns into the dominant attractor of the system for $Q \gtrsim 0.4$.

We note that the four regimes described above are reached by changing only the coupling strength $Q$ and can be found in a broad parameter range. Furthermore, the system frequently exhibits a strong multistability, which is an ongoing issue in the discussion of addressable memory in genetic networks and other information processing systems. The determined dynamical regimes and the multistability are stable and persist in a noisy environment. For instance, protein fluctuations larger than $25 \%$ of their mean level do not alter the clustering attractor in the multistable parameter range.

We have proposed the integration of two paradigms in synthetic biology, namely, the repressilator circuit [3] and a cell-to-cell communication module based on quorum sensing [15], combined in such a way that phase-repulsive coupling arises among the genetic oscillators. The theoretical predictions reported here are amenable to observation at the single-cell level via time-lapse fluorescence microscopy [16]. This technique is very useful to experimentally test theoretical predictions in genetic networks [25]. Beside its biological consequences, the model sheds light on new phenomena from a general nonlinear dynamics viewpoint. First, the oscillation death (OD) evidenced in the present work is stable far from any Hopf bifurcation in a wide range of the phase diagram. This contrasts with previous observations $[21,24,26]$, where OD occurred only in a small range close to a Hopf bifurcation. Second, we could demonstrate a combination of OD and IHLC in a large globally coupled system, which leads to a nontrivial clustering. Third, the phase-repulsive coupling leads to multistability between the regimes of OD, IHLC, and the single fixed point. The many possible dynamical regimes available to the cellular population improve its adaptability and robustness. Such an improved efficiency induced by coupling can probably exist in natural genetic networks and can definitely be exploited in synthetic devices. We expect that our theoretical findings will stimulate further investigations, e.g., on the influence of diversity in this regime. The results may also be relevant for other systems with repressive coupling, which leads to strong competition between the members of large ensembles.

E. U. acknowledges financial support from the Alexander von Humboldt Foundation, A.Z. from VWfoundation and BSC through HPC EU program, E.I.V. from the Program "Radiofizika" Russian Academy and RFBR Grant No. RFBR 05-02-16518, and J. G.-O. from the Ministerio de Educación y Ciencia (Spain) (project ORDEN), from the Generalitat de Catalunya, and from the European Commission (project GABA, FP6-NEST Contract No. 043309).

[1] J. Hasty et al., Nature (London) 420, 224 (2002).

[2] D. Sprinzak and M. B. Elowitz, Nature (London) 438, 443 (2005).

[3] M. Elowitz and S. Leibler, Nature (London) 403, 335 (2000).

[4] M. B. Elowitz (private communication).

[5] J. García-Ojalvo et al., Proc. Natl. Acad. Sci. U.S.A. 101, 10955 (2004).

[6] E. I. Volkov and M. N. Stolyarov, Phys. Lett. A 159, 61 (1991).

[7] S. K. Han et al., Phys. Rev. Lett. 75, 3190 (1995).

[8] G. Balázsi et al., Phys. Rev. E 64, 041912 (2001).

[9] H. Meinhardt, Models of Biological Pattern Formation (Academic, New York, 1982).

[10] R. Laje and G. B. Mindlin, Phys. Rev. Lett. 89, 288102 (2002).

[11] W. Metzner, J. Neurosci. 13, 1862 (1993).

[12] L. Glass and M.C. Mackey, From Clocks to Chaos: The Rhythms of Life (Princeton University Press, Princeton, NJ, 1988).

[13] M. R. Atkinson et al., Cell 113, 597 (2003).

[14] D. McMillen et al., Proc. Natl. Acad. Sci. U.S.A. 99, 679 (2002).

[15] L. You et al., Nature (London) 428, 868 (2004).

[16] N. Rosenfeld et al., Science 307, 1962 (2005).

[17] L.A. Moran et al., Biochemistry (Neil Patterson Publishers/Prentice Hall, Englewood Cliffs, NJ, 1994), 2nd ed.

[18] J. M. Raser and E. K. O'Shea, Science 309, 2010 (2005).

[19] D. Golomb et al., Phys. Rev. A 45, 3516 (1992).

[20] K. Kaneko and T. Yomo, Physica (Amsterdam) 75D, 89 (1994).

[21] W. Wang et al., Chaos 10, 248 (2000).

[22] J. Tyson and S. Kauffman, J. Math. Biol. 1, 289 (1975).

[23] E. I. Volkov and V. A. Romanov, Phys. Scr. 51, 19 (1995).

[24] A. Kuznetsov et al., SIAM J. Appl. Math. 65, 392 (2004).

[25] G. M. Süel et al., Science 315, 1716 (2007).

[26] R. Herrero et al., Phys. Rev. Lett. 84, 5312 (2000). 